\title{
POWER FREQUENCY OVERVOLTAGES GENERATED BY SOLAR PLANT INVERTERS
}

\author{
F.J. Pazos ${ }^{1}$, A. Barona ${ }^{2}$, J. Amantegui ${ }^{1}$, E. Azcona ${ }^{1}$ and S. Fernández ${ }^{1}$ \\ ${ }^{1}$ Iberdrola Distribución Eléctrica \\ Avenida San Adrián 48 - 48003 Bilbao (Spain)
}

e-mail: fi.pazos@iberdrola.es, javier.amantegui@iberdrola.es, eduardo.azcona@iberdrola.es, $\underline{\text { ffenandez@iberdrola.es }}$

\author{
${ }^{2}$ Iberdrola Distribución Eléctrica \\ Menorca 19 - 46023 Valencia (Spain) \\ e-mail: abarona@iberdrola.es
}

\begin{abstract}
.
A new kind of overvoltage, not covered by present standards, has been detected in solar plants when switching the inverters off. This power frequency overvoltage can be transmitted to the MV network, giving rise to damages to some pieces of low voltage electronic equipment.

This phenomenon has been investigated in the field, testing several inverters in different solar plants, where the overvoltages, as well as the damages in revenue meters, have been reproduced and recorded.

It has been proved that some inverter configurations are more prone to create severe overvoltages than other. In addition, some solutions have been tested and a laboratory test to reproduce the phenomenon is proposed.
\end{abstract}

\section{Key words}

Solar plant, photovoltaic, overvoltage, islanding, electronic appliance damage, standards

\section{Introduction}

In the last years, damages in electronic equipment of photovoltaic plants have been reported. Quite often, these damages affected revenue meters, which usually were replaced without further investigation. However, the placement into service of large solar plants and the corresponding increase of damaged devices have made clear the need of a more detailed assessment.

Thus, it was discovered that damages take place during a switch upstream of the inverter (whether low or high voltage level), in such way that one or several inverters become isolated from the rest of the grid, according to the simplified scheme shown in figure 1 .

At the field tests, the complete phenomenon -switch opening followed by revenue meter damage- was easily reproduced. Measurements proved that the opening of any of the switches upstream of the inverter gives rise to an important overvoltage, as can be seen in figure 2 .
However, some aspects have to be taken into consideration.

All the inverters comply with present standards, since none of the existing standards deals with this event.

Although all the tested inverters produced some overvoltages, not all of them were high enough to damage meters. Clear differences could be observed between different brands.

Not only meters, but other electronic devices suffered damages. Meters were more prone to be damaged simply because in large photovoltaic plants they are usually the only electronic device present other than the inverter.

\section{Analysis}

Figure 2 shows the wave-form of voltages and currents, during the opening of a Low Voltage switch that isolates two $25 \mathrm{~kW}$ inverters, according to the simplified scheme of figure 1(the two parallel inverters are represented as only one).

It can be observed that the inverter trips at $60 \mathrm{~ms}$ but, until then, there is a severe overvoltage up to about twice the nominal voltage. This overvoltage appears when the inverter feeds and over-excites the inverter transformer and the ancillary services transformer.

Figure 3 and 4 shows the switching of the $20 \mathrm{kV}$ circuit breaker, which disconnects four $100 \mathrm{~kW}$ inverters. In this case, the inverters keep on energizing 50 transformers, $100 \mathrm{kVA}$ each; giving rise, equally, to an overvoltage.

In this event, the overvoltage is transferred to $\mathrm{MV}$, as can be observed in figure 5, which represents the voltage in the secondary of an unloaded $20 / 0.4 \mathrm{kV}$ transformer. This overvoltage means an excessive stress for other electronic device power supplies, such as revenue meters. 


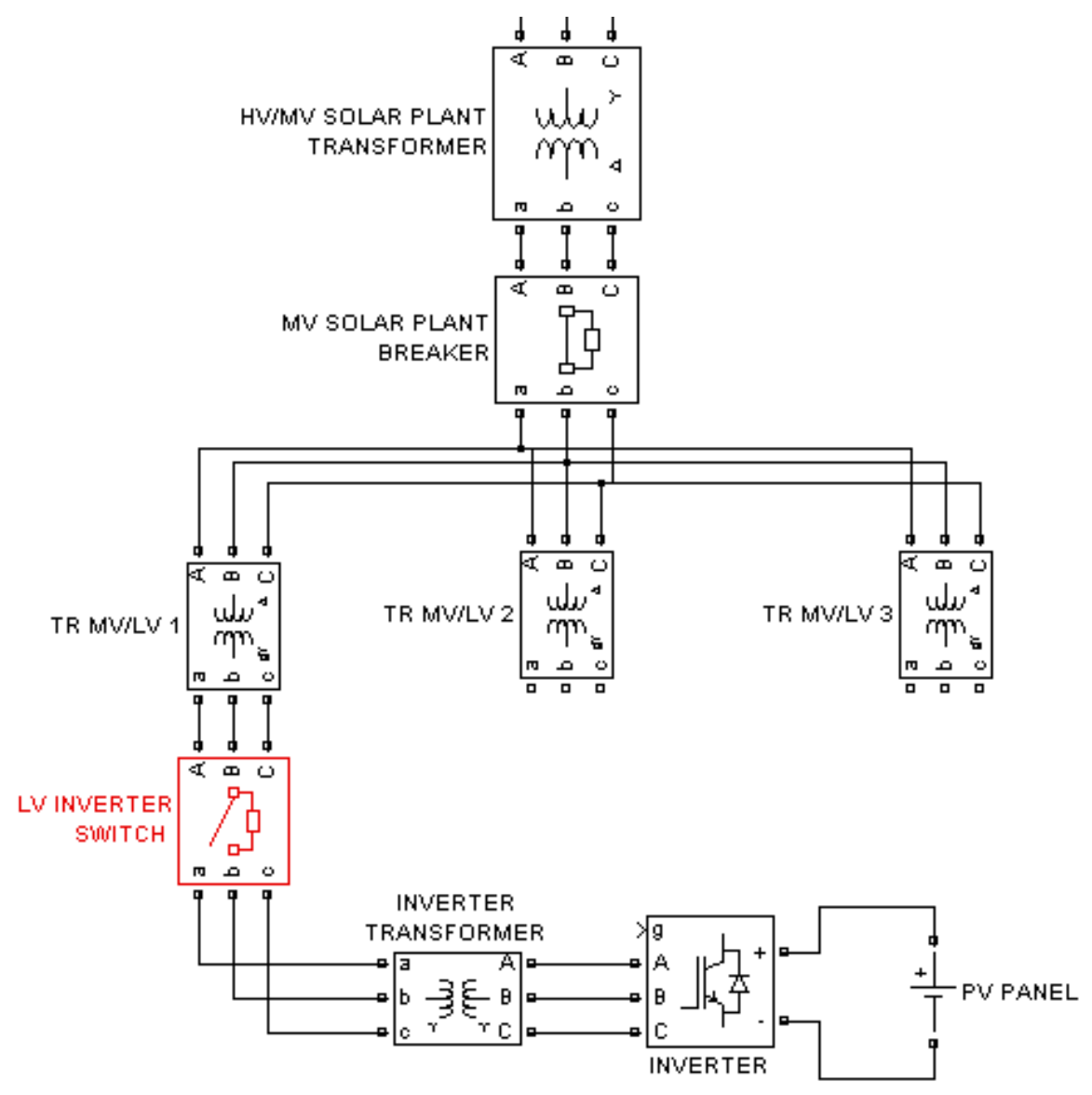

Figure 1: Simplified scheme of a photovoltaic plant

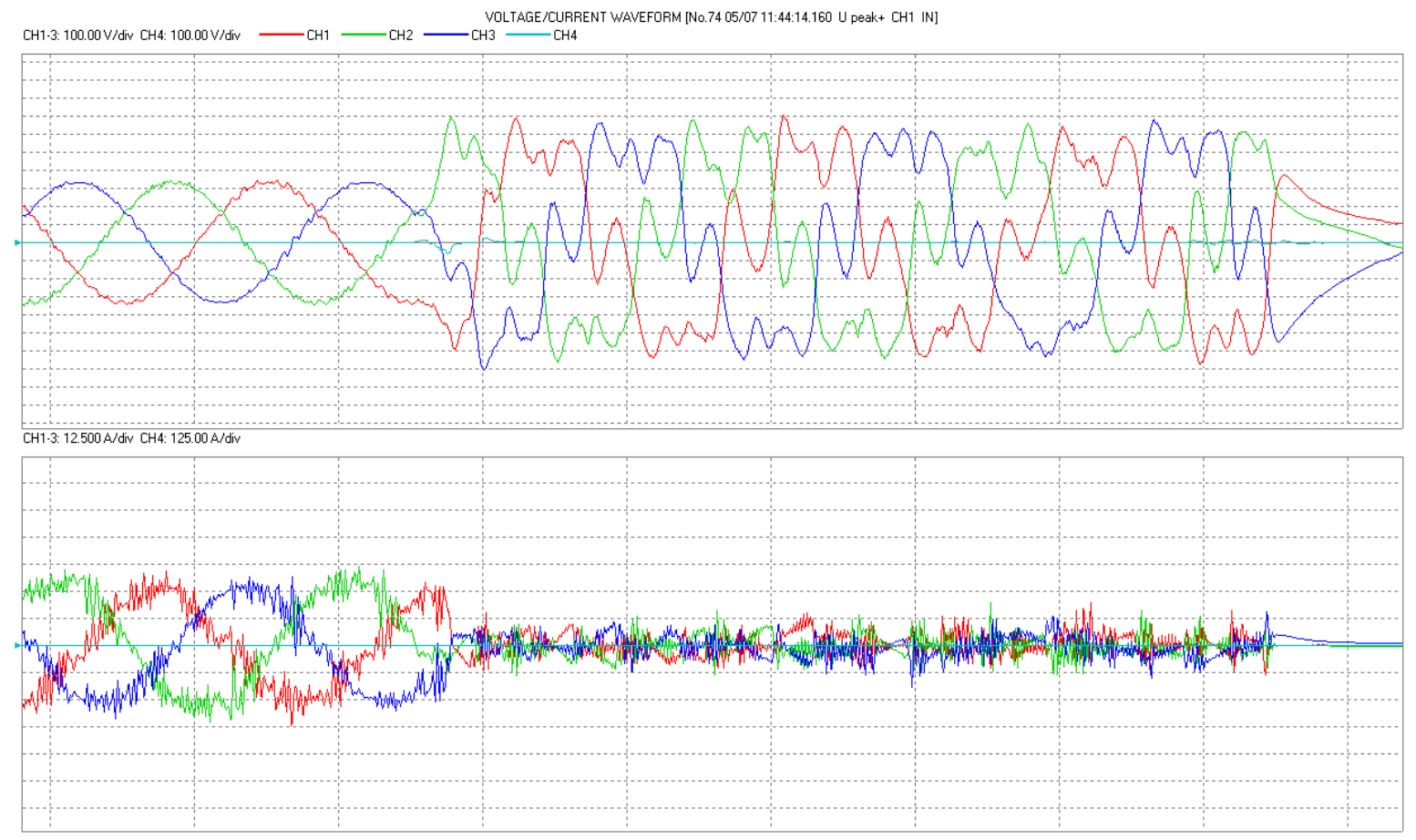

Figure 2: Overvoltage leading to revenue meter damage during LV switching-off (voltages and currents) 


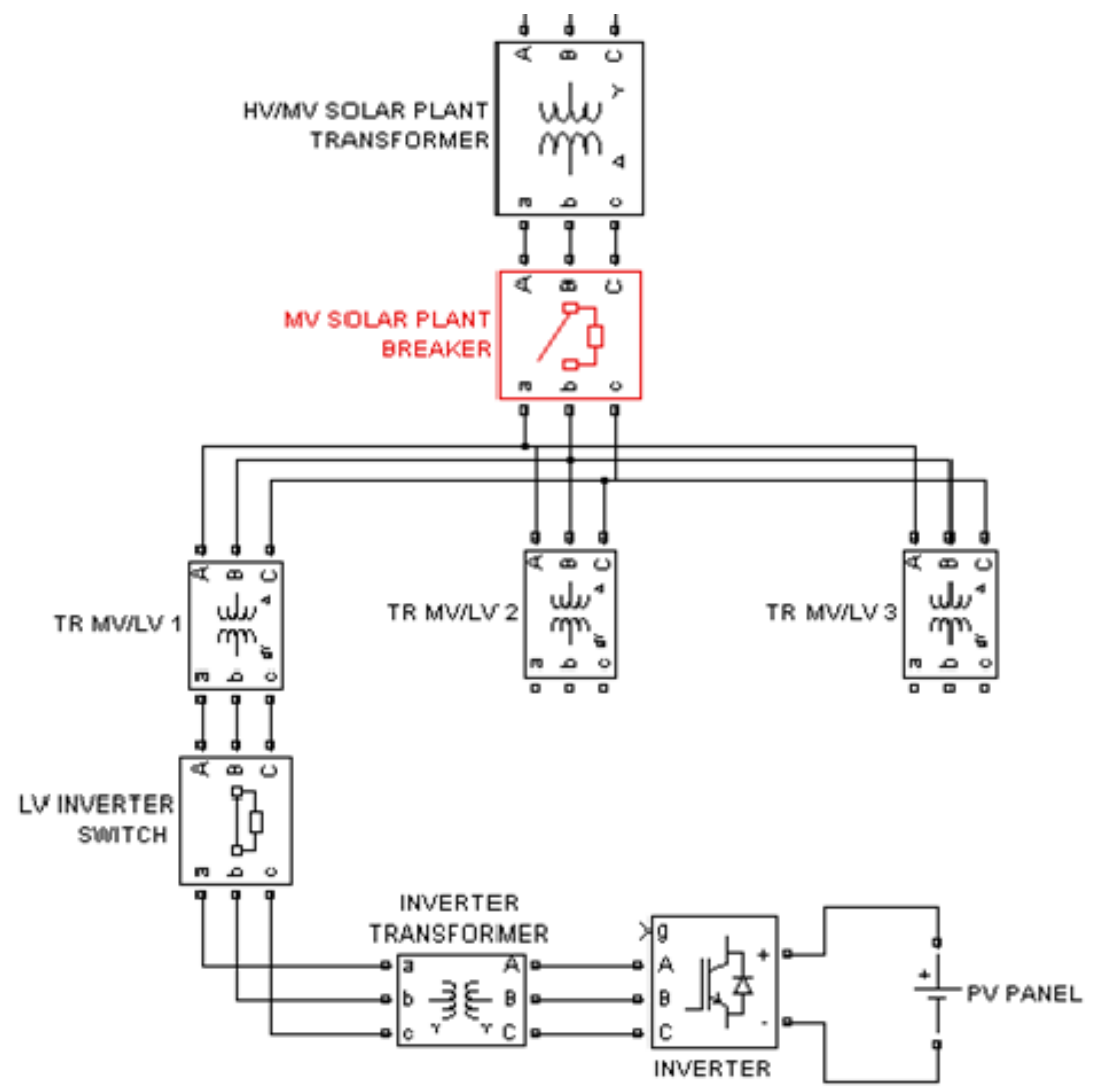

Figure 3: MV Switching-off
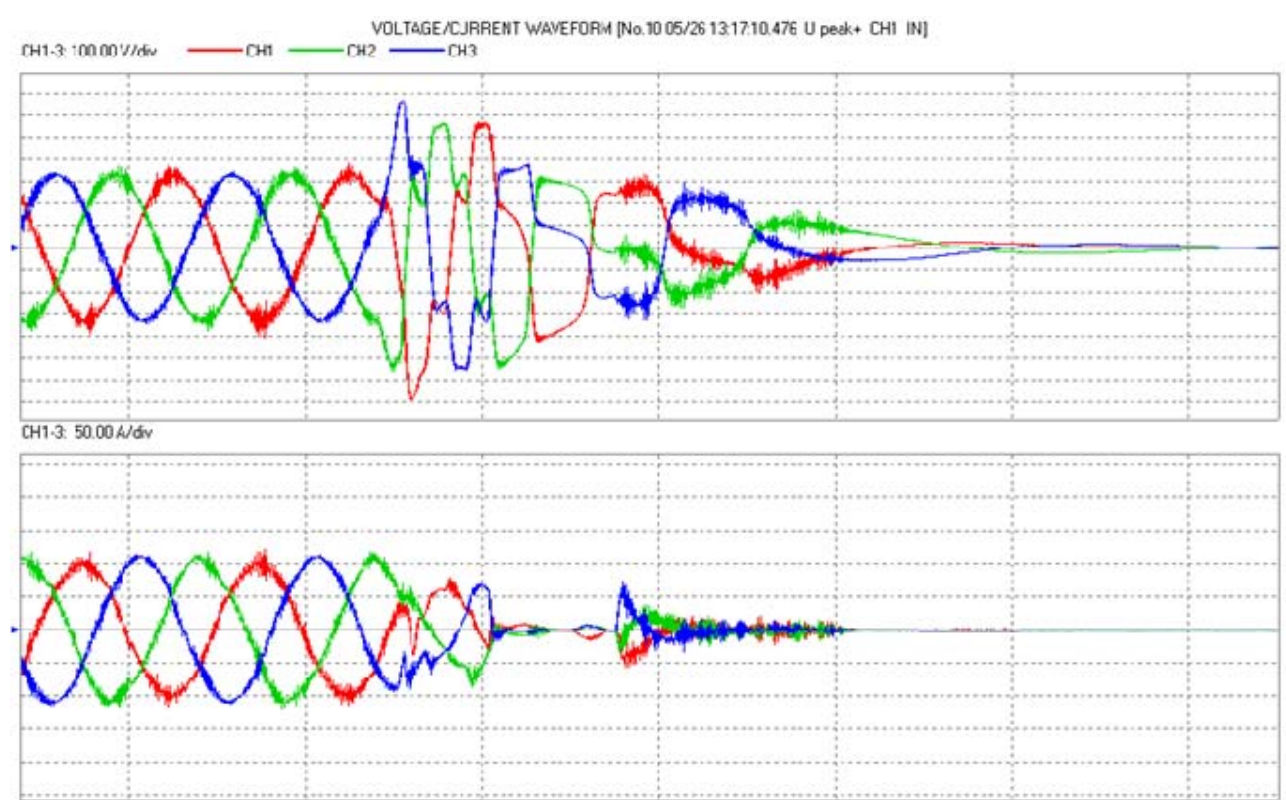

Figure 4: Overvoltage in LV side of TR MV/LV1 due to MV Switching-off

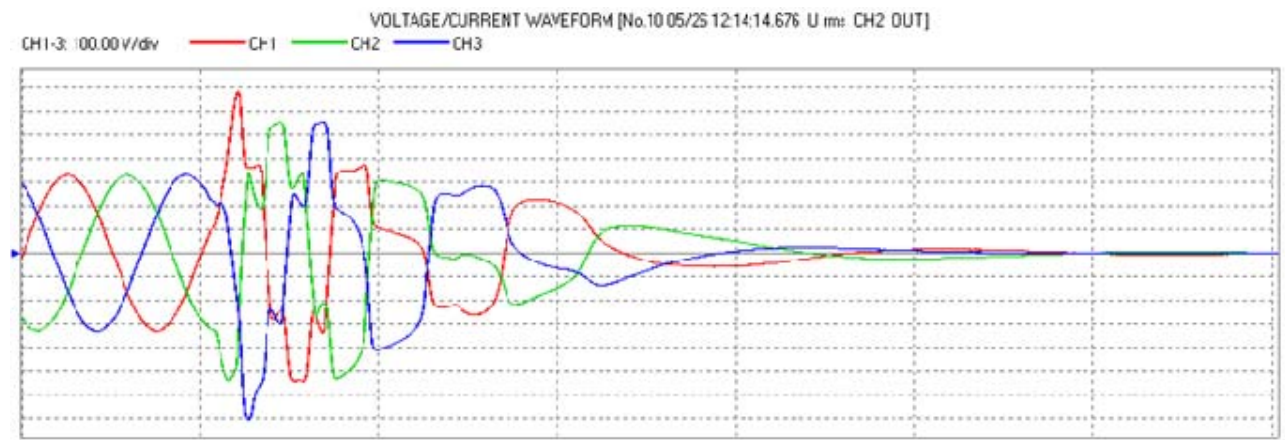

Figure 5: Overvoltage transmitted from TR MV/LV1 to TR MV/LV2. 
The stress for other electronic devices can be observed in figure 6 which represents the result of the simulation of a three phase rectifier behaviour facing one of the measured overvoltages. It can be noticed a huge voltage increase in the dc bus and an excessive inverse voltage applied to the diodes. These values are high enough to provoke many electronic devices to damage.
Besides, these high values give rise to the intervention of surge protection, namely varistors in LV and arresters in MV. Depending on their nominal rate, the exposure of these devices to long overvoltages can lead to their destruction, with the consequent short-circuit in the LV, MV or even HV networks.
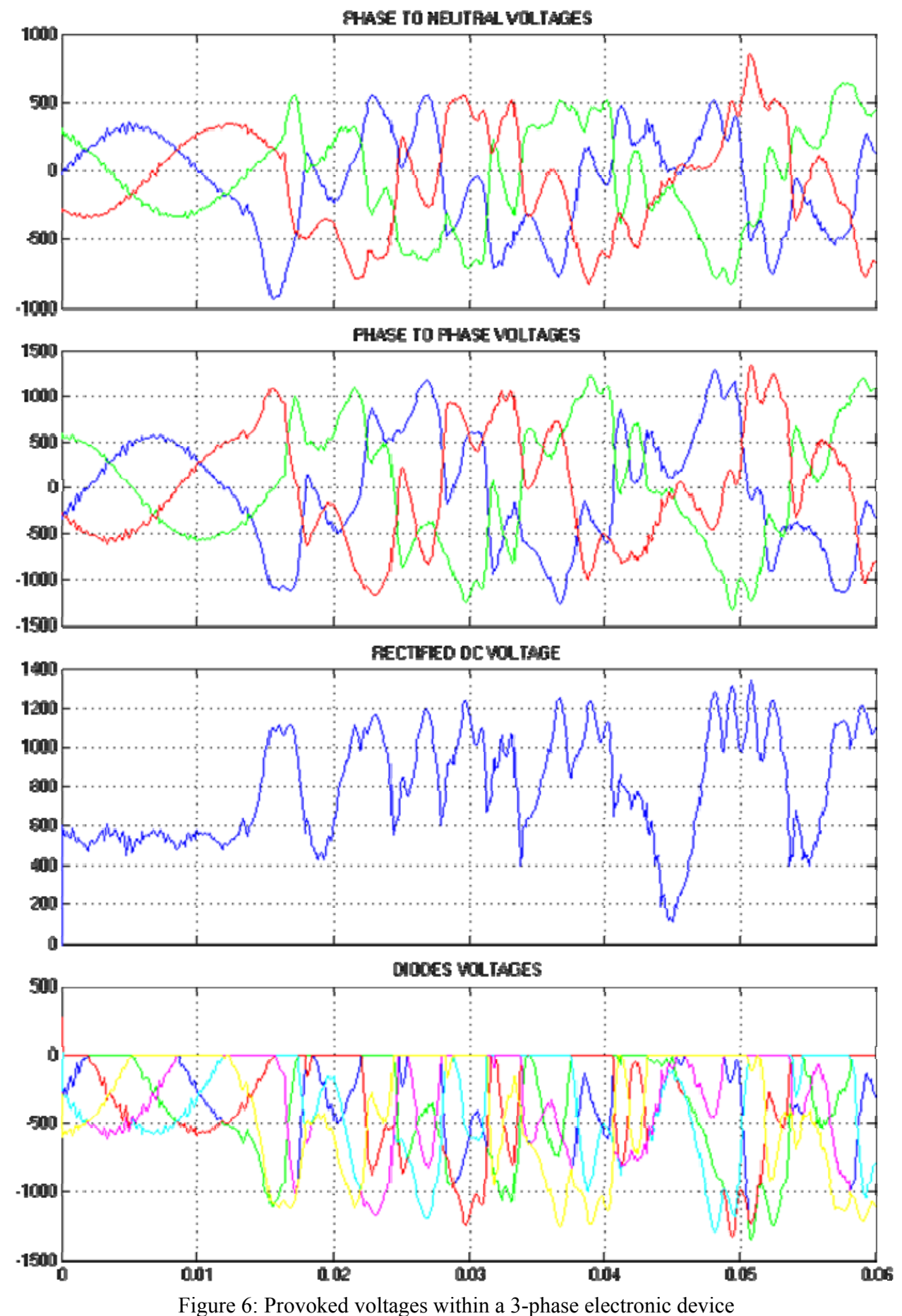
In most cases, the most relevant overvoltage is phase-tophase. However, extremely severe phase-to-neutral overvoltages have been found as can be seen in figure 7 . In this case, peak values up to $1.17 \mathrm{kV}$ (358\% of nominal peak value) have been measured.

In addition, the tested inverters included surge suppressors, connected phase-to-neutral and ranging from
300 to 320 Vrms. Since it is necessary that at least two of them conduct, their residual voltages are added and the phase-to-phase voltage is scarcely reduced. Figure 8 shows the effect of such protections, rated for a 400/230 $\mathrm{V}$ network. It can be seen that, although the varistors clamp slightly the voltage, the peak values phase-tophase still exceed $1200 \mathrm{~V}$, that can still be over the withstanding limit of many devices.

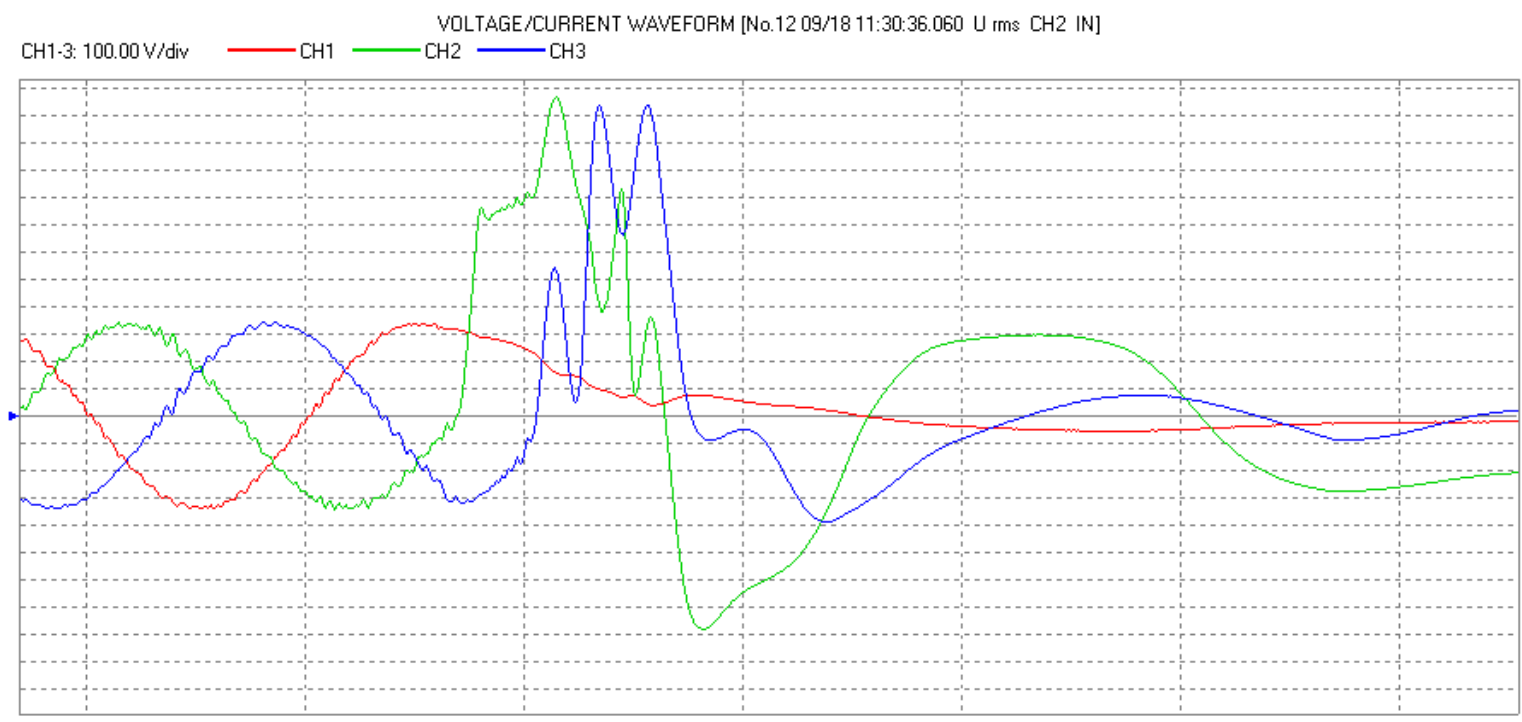

Figure 7: Severe phase-to-neutral overvoltage

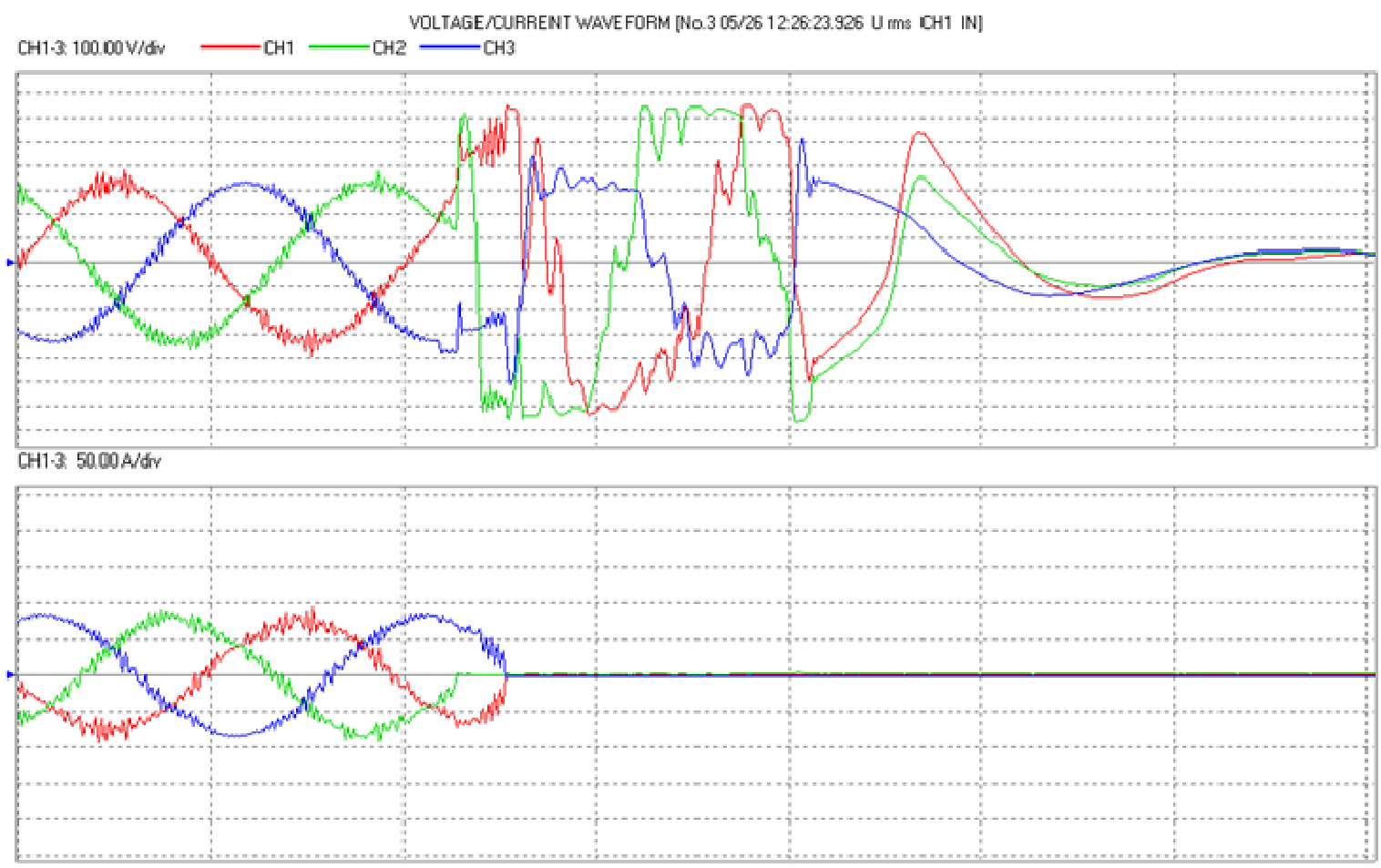

Figure 8: Effect of surge protections connected phase-to-neutral 


\section{Overvoltage generation test for solar inverters}

The following procedure has been proposed to inverter manufacturers, in order to reproduce the circumstances in which overvoltages have been measured in solar plants, when the solar system becomes isolated.

1. Install two inverters in parallel, fed through a low voltage switch, according to the scheme of figure 9.

- The transformers are the internal ones for galvanic isolation. In case that the inverters do not use internal transformers, external transformers shall be installed.

- Downstream the switch, there is only the solar system, with no other load.

- Depending on the inverter type, in some cases the phenomenon can be reproduced with only one inverter, although for completion's sake, the test must be performed with, at least, two inverters.

- Install a voltage recorder downstream the switch, measuring the voltage in the side that becomes isolated from the grid. The recorder must have a minimum sampling frequency of $10 \mathrm{kHz}$, and shall record:
- The 3 phase-to-ground voltages.

- The 3 phase-to-phase voltages. Taking into account that these voltages can be higher that 800 Vrms, which could be excessive for many recorders, it would be admissible to calculate the phase-to-phase voltage from the phase-to-neutral voltage, by subtracting sample by sample (e.g. $\mathrm{V} 12=\mathrm{V} 1-\mathrm{V} 2$ ).

2. Connect the two inverters at $10 \%$ of their nominal power.

3. Open the switch and record the waveform, calculating the maximum instantaneous voltage (peak value) phase-to-neutral (or ground) and phase-to-phase.

4. The overvoltage duration must be measured as the time between the moment when the voltage, in any of the three phases, exceeds the peak nominal voltage limits (i.e. $230 x \sqrt{2}+10 \%$ or $400 x \sqrt{2}+10 \%$ ) until the values of any of the three phases are permanently within the nominal limits.

5. Repeat the test at $50 \%$ and $100 \%$ of nominal power.

By following this procedure, or even a simplification of it, the affected manufacturers have been able to reproduce repetitively the overvoltages without putting the solar plants at risk.

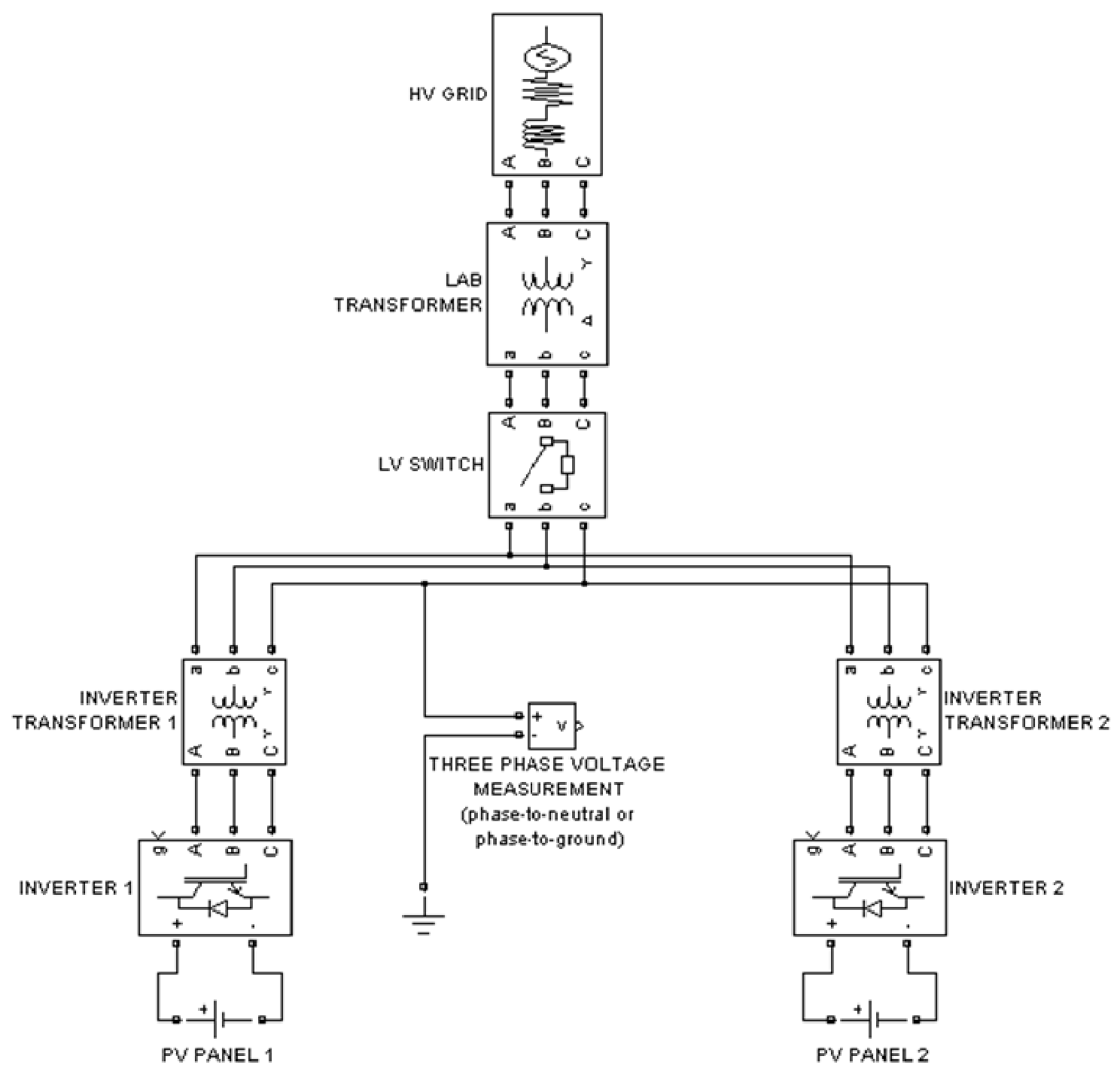

Figure 9: Overvoltage generation test 


\section{Tested solutions}

In order to minimize the overvoltages some solutions have been tested.

\section{A. Software protection}

The duration of the overvoltage shown in the previous figures is too long to be treated with surge suppressors, such as varistors, since they could not withstand the energy that they should dissipate.

Therefore, the first measure to be taken is to include a.c. voltage supervision, in order to limit the overvoltage duration. This supervision should stop the inverter switching in the very moment when the beginning of the overvoltage is detected.

Figure 10 shows the behaviour of this type of protection already incorporated in an inverter. As can be noticed, it limits both the duration and the magnitude of the overvoltage.

Although the behaviour in this case is much better that in the preceding ones, it can be seen that there are still overvoltages. In this event, the phase-to-phase voltage, the d.c. voltage or the inverse voltage at a diode would reach a peak of approximately $1100 \mathrm{~V}$.
Faster protection reduces the overvoltage to lower values. But even with detections of 1 millisecond, they have been measured peak voltages phase-to-phase of $915 \mathrm{~V},(162 \%$ of the nominal voltage), while the most affected phaseto-neutral voltage reaches a $188 \%$ of the nominal voltage.

\section{B. Surge suppression}

The persisting overvoltages, despite the fast switching stop, could be controlled by means of surge suppressors.

Nevertheless, they have been proved inefficient when connected phase-to-neutral. Given that the inverter behaviour is three-wire and does not requires the neutral, surge suppressors would be more efficient if they were connected phase-to-phase, i.e. in delta connection, since this would allow lower residual voltages. As an example, in the test illustrated by figure 11, the surge suppressors reduced the phase-to-phase peak voltage by a $30 \%$.

However, in order to use these suppressors safely, it is necessary to dimension them according to the energy delivered by each inverter. Therefore, the manufacturers should specify them taking into account the total power of the inverter and the tripping time of their overvoltage protection.

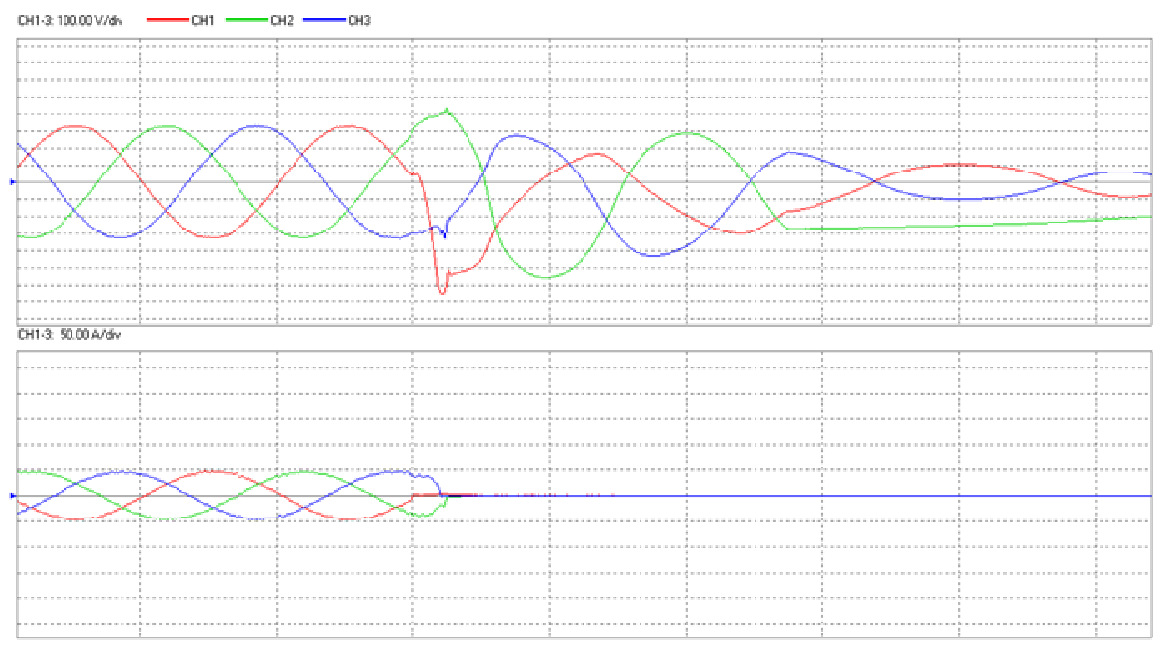

Figure 10: behaviour with fast software protection
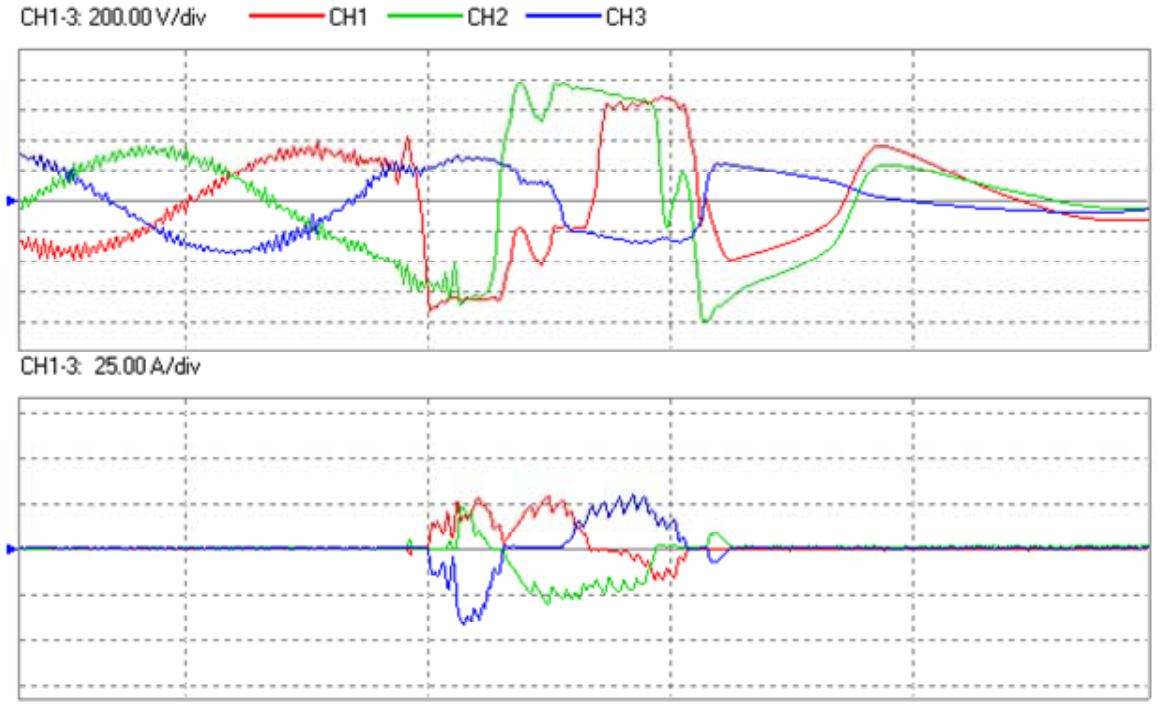

Figure 11: Use of phase-to-phase surge suppressors.

Phase-to-neutral voltages and surge suppressor currents. 

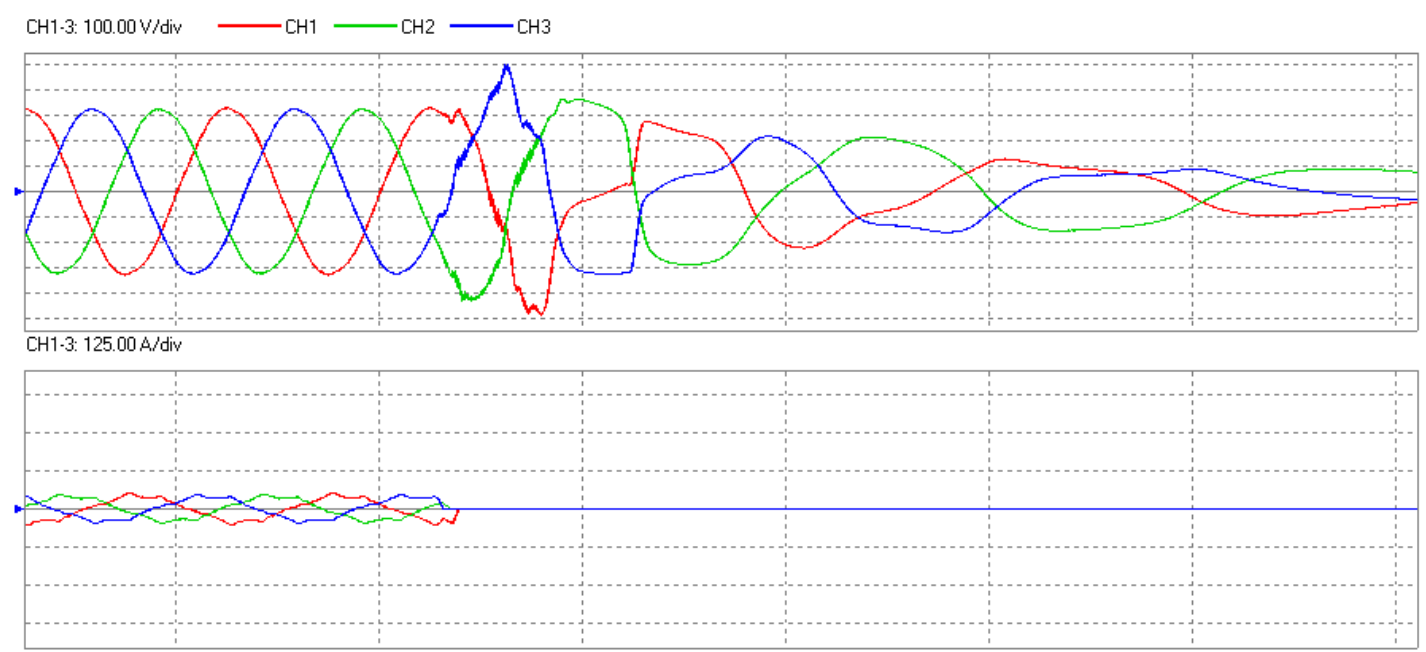

Figure 12: Behaviour of an inverter with dc/dc chopper

In addition, the overvoltage software protection settings should be lower than the surge suppressor residual voltage, in order to guarantee the switching tripping while the suppressors are working.

In those cases that surge suppressors were used, the maintenance protocols should take into account that this kind of protection requires revision and, when they get to their end of life, needs replacement.

Besides, the inverter operation should be dependent on the good state of the surge suppression system. To this aim, both suppressors and their circuit breaker should have auxiliary contacts to stop the inverter in case of failure.

\section{Inverters with DC/DC Chopper}

The inverter behaviour depends on its configuration. The aforementioned measurements correspond to inverters whose dc busbar do not have a fix voltage, since it varies depending on the insolation.

Nevertheless, some inverters include a $\mathrm{dc} / \mathrm{dc}$ chopper to control and limit its dc voltage. Figure 12 shows the switching-off of an inverter using this converter. As can be seen, the resulting overvoltage is the lowest of the tested inverters, with a peak value of $134 \%$.

\section{Conclusions}

Severe overvoltages have been detected in solar plants when switching the inverters off. These overvoltages damaged some pieces of low voltage equipment, in particular revenue meters and electronic devices associated with the inverters. The overvoltages take place during a switch upstream of the inverter (whether low or high voltage level), in such way that one or several inverters left isolated from the rest of the grid.

On the other hand, in large solar plants (several MW) fed by Medium Voltage busbars with no other load, it has been verified that overvoltages in the MV side reach dangerous values. Given that all systems are interconnected through their transformers, such overvoltages affect not only the low voltage equipment, but the medium or high voltage grid.

This problem is not covered by present standards, since usual limits are not oriented towards transients but towards relatively long overvoltages during normal operation.

The phenomenon is related to the interaction among the inverters until the anti-islanding protection trips. It must be pointed out that these islanding conditions are not considered either in the standards.

In any event, the problem is not the islanding detection, whose procedures are usually focused on a reliable detection when generation and consumption are balanced. The circumstances in which the overvoltage appears are the opposite, with a generation much higher than the load.

Since standards are not applicable, it has been necessary to investigate this phenomenon in the field, testing several inverters, from different manufacturers, in different solar plants. The overvoltages, as well as the damages in meters, have been reproduced and recorded. In addition, a simplified test method has been proposed. The affected manufacturers have successfully used this method, or a simplification of it, to reproduce the problem in laboratory.

It has been proved that some inverter configurations are more prone to create severe overvoltages than other.

Thus, the use of $\mathrm{dc} / \mathrm{dc}$ choppers is a possible solution to limit the overvoltage.

For those inverter configurations that generate overvoltages, other solutions have been tested:

- Overvoltage supervision, focused on the a.c. side, to rapidly stop the inverter switching in case of overvoltage.

- Particularly rated surge suppressors to reduce the voltage to peak values within the admissible limits for all the equipment connected to the grid.

Although these solutions do not provide an optimum overvoltage value, they can reduce significantly the damage risk, being applicable to existing equipment.

Present EMC standards, basically oriented towards loads, do not cover the event of overvoltage generation. For this reason, there are not available neither emission limits nor laboratory tests.

Therefore, further work seems essential, especially to develop appropriate standards and tests to be applied to distributed generation. 